Eur Heart J Acute Cardiovasc Care. 2016 April ; 5(2): 108-116. doi:10.1177/2048872615584079.

\title{
Outcomes in patients undergoing percutaneous ventricular assist device implantation for cardiogenic shock
}

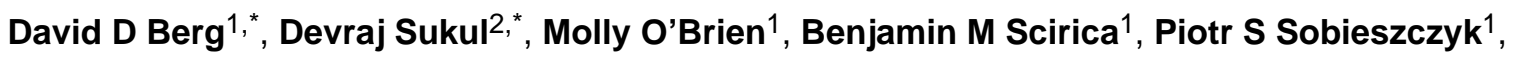 \\ Benjamin A Olenchock ${ }^{1}$, Erin A Bohula ${ }^{1}$, and David A Morrow ${ }^{1}$ \\ ${ }^{1}$ Brigham and Women's Hospital, Harvard Medical School, USA \\ 2University of Michigan Medical School, USA
}

\begin{abstract}
Background-Percutaneous ventricular assist devices (PVADs) offer an important but resourceintensive option for management of severe cardiogenic shock (CS). Optimal selection of patients for PVAD support remains undefined. We investigated outcomes, including characteristics associated with in-hospital survival, during PVAD support for CS.
\end{abstract}

Methods-We established a prospective quality improvement program among patients undergoing TandemHeart PVAD implantation for CS at Brigham and Women's Hospital (Boston, MA). We evaluated 65 consecutive patients between 2006 and 2014, analyzing demographic, clinical, laboratory, hemodynamic, and survival data.

\begin{abstract}
Results-Thirty-two patients (49.2\%) survived to hospital discharge, of which 12 received destination surgical therapy. Baseline characteristics associated with survival included younger age ( $47 \pm 15$ years vs $61 \pm 11$ years; $p<0.001)$, non-ischemic cardiomyopathy (NICMP) vs ischemic CMP (survival 70.4\% vs $34.2 \%, p=0.004$ ), and, paradoxically, lower presenting left ventricular ejection fraction (LVEF) (survival $66.7 \%$ for LVEF $₫ 5 \%, 41.2 \%$ for LVEF $16-25 \%$, $25.0 \%$ for LVEF $>25 \% ; p=0.010)$. Younger age $(p=0.026)$ and NICMP $(p=0.034)$ remained independent predictors of survival. Twenty-four hours after PVAD placement, a more modest increase in cardiac index $\left(\unlhd 0.75 \mathrm{~L} / \mathrm{min} / \mathrm{m}^{2}\right.$ ) was associated with higher in-hospital mortality (OR $6.3,95 \%$ CI 1.8-22.1), as was lack of improvement in serum anion gap ( $\_$mEq/L; OR 5.1, 95\% CI 1.6-16.6).
\end{abstract}

Conclusions-Despite intensive care and provision of circulatory support, survival is poor in severe CS. Patients in CS with younger age and NICMP were more likely to survive to hospital discharge. Less robust hemodynamic improvement and persistent acidosis after 24 hours of PVAD support also identified patients less likely to survive.

Reprints and permissions: sagepub.co.uk/journalsPermissions.nav

Corresponding author: David A Morrow, Levine Cardiac Intensive Care Unit, Cardiovascular Division, Brigham and Women's Hospital, 75 Francis Street, Boston, MA 02115, USA. dmorrow@partners.org.

These authors contributed equally to the development of this manuscript.

Conflict of interest

None declared. 


\section{Keywords}

Shock; cardiomyopathy; mechanical circulatory support; ventricular assist device; cardiovascular critical care

\section{Introduction}

Cardiogenic shock (CS), the most severe form of acute heart failure, is characterized by endorgan hypoperfusion resulting from impaired cardiac output. ${ }^{1}$ Rapid reversal of end-organ hypoperfusion is critical to preventing the onset of multi-organ dysfunction and improving patient survival. ${ }^{2,3}$

Mechanical circulatory support (MCS) devices provide a therapeutic option for patients with cardiogenic shock refractory to pharmacologic treatment. The most widely available and utilized MCS device is intra-aortic balloon pump (IABP) counterpulsation; ${ }^{4}$ however, IABP support has not been shown to be beneficial when evaluated in an era of early revascularization and optimal medical therapy. ${ }^{5}$ Therefore, interest in percutaneous ventricular assist devices (PVADs) that offer greater hemodynamic support and the potential for greater clinical benefit has continued.

The TandemHeart PVAD is a percutaneously placed, intravascular, continuous flow, MCS device that can be placed in the cardiac catheterization laboratory, and is capable of providing up to $5.0 \mathrm{~L} / \mathrm{min}$ of cardiac output. ${ }^{6.7} \mathrm{In}$ this system, oxygenated blood is withdrawn from the left atrium (LA) by a catheter placed in a central vein (usually via a femoral approach) which reaches the LA by atrial septostomy. The oxygenated blood is then propelled through a continuous flow centrifugal pump into the abdominal aorta or iliac artery via a femoral arterial catheter. Right ventricular support is also possible by percutaneous placement of the inflow cannula in the right atrium and the outflow catheter into the pulmonary artery. As such, two PVADs may be used concurrently to provide biventricular support. ${ }^{6,7}$ Compared with an IABP, the TandemHeart has been shown to provide greater hemodynamic support in animal models as well as in patients with CS. ${ }^{8}$ Despite their ability to reverse hemodynamic collapse, PVADs are resource-intensive and have not been evaluated in studies of sufficient sample size to determine whether they can improve clinical outcomes in severe CS. Optimal selection of patients for PVAD support remains undefined and is likely to be important to appropriately allocate resources and optimize outcomes. We investigated the clinical outcomes and patient characteristics associated with in-hospital survival among patients receiving PVAD support for CS in a large, tertiary care, academic medical center.

\section{Methods}

\section{Patient selection}

We established a prospective quality improvement program among patients undergoing PVAD implantation for CS at Brigham and Women's Hospital in Boston, MA. A team of critical care cardiologists, heart failure specialists, and interventional cardiologists determined candidacy for PVAD implantation when a patient demonstrated progressive or 
persistent CS despite pharmacologic support, IABP counterpulsation, and/or Impella, and was deemed to have a potential treatment destination (e.g. recovery, surgery, cardiac transplantation). CS was defined by both severe hemodynamic impairment (systolic blood pressure $<90 \mathrm{mmHg}$ and cardiac index $<2.2 \mathrm{~L} / \mathrm{min} / \mathrm{m}^{2}$ or the need for inotropic or vasopressor support) and at least one indicator of end-organ hypoperfusion, which included altered mental status, creatinine increase of $>0.3 \mathrm{mg} / \mathrm{dL}$ or at least $50 \%$ of baseline, urine output $<0.5 \mathrm{~mL} / \mathrm{kg} / \mathrm{hr}$ for $>6$ hours, or serum alanine aminotransferase, aspartate aminotransferase, or total bilirubin levels greater than twice the upper limit of normal.

Following PVAD placement, all patients received care in the cardiac intensive care unit. Decisions regarding the amount of assisted cardiac output provided by the PVAD and additional pharmacologic support (e.g. vasopressors and inotropes) were made according to our institutional standards.

\section{Data collection}

We collected demographic, clinical, laboratory, and hemodynamic data as part of routine care. Patients were classified as having an ischemic cardiomyopathy (ICMP) based on a history of prior coronary revascularization, history of prior myocardial infarction (MI), or evidence of obstructive coronary artery disease ( $270 \%$ stenosis in at least one major epicardial coronary artery) on cardiac catheterization. Patients who did not meet the criteria for ICMP were classified as having a non-ischemic cardiomyopathy (NICMP). In rare circumstances, patients were classified as having NICMP when the degree of myocardial dysfunction was determined to be out of proportion to the burden of coronary artery disease based on case review. NICMP was classified as dilated or hypertrophic using echocardiographic criteria. The etiology of NICMP was categorized as idiopathic, familial, infectious, autoimmune, valvular, toxic/metabolic, or pulmonary vascular based on available clinical data. Patients were also classified as having left ventricular, right ventricular, or biventricular failure based on available echocardiographic and hemodynamic assessments.

Baseline laboratory data were obtained within 24 hours prior to the initiation of PVAD support, and follow-up laboratory data were obtained $24( \pm 12)$ hours after PVAD placement. Hemodynamic data were collected immediately prior to PVAD implantation, and repeated at $3( \pm 3)$ hours and $24( \pm 12)$ hours after PVAD placement. The hemodynamic parameters included in the analysis were cardiac index (CI), mean arterial pressure (MAP), right atrial pressure (RAP), pulmonary capillary wedge pressure (PCWP), and the combined number of inotropes and vasopressors used. Pulmonary arterial catheter monitoring was available for $100 \%$ of patients included in the analysis. CI was obtained primarily using the Fick method. When Fick calculations were unavailable, either continuous cardiac output monitoring or standard thermodilution techniques were used. The total number of units of packed red blood cells (PRBC) given to each patient from the time of PVAD placement through 48 hours following PVAD removal, or until the time of any major surgery (intra-abdominal or intra-thoracic), whichever came first, was recorded.

Vital status was obtained for all patients at the time of discharge and at six months following initiation of PVAD support. Patients were classified as being "bridged to surgery" when they received cardiac surgical therapy including valve repair/replacement, atrial or 
ventricular septal defect repair, coronary artery bypass grafting, surgical ventricular assist device placement, and/or cardiac transplant during the index hospitalization, and survived to discharge. All patients who survived to hospital discharge without surgical intervention were defined as being "bridged to recovery." Patients who died before hospital discharge were categorized as "in-hospital deaths", regardless of whether they underwent surgery.

\section{Statistical analysis}

We compared baseline demographic, clinical, laboratory, and hemodynamic characteristics between patients who survived and died prior to hospital discharge using the Fisher's exact or chi-square test for categorical variables and the Student $t$ test or Mann-Whitney U test for continuous variables. We evaluated changes in hemodynamic parameters after three hours and 24 hours of PVAD support for all patients using Wilcoxon Signed Rank testing. Multivariable analysis was performed using logistic regression. The following variables were assessed: age, sex, BMI, type of cardiomyopathy (ICMP vs NICMP), type of ventricular dysfunction ( $\mathrm{LV}$ vs RV vs BiV), presenting $\mathrm{LV}$ ejection fraction, preceding cardiac arrest, preceding IABP support, preceding Impella support, transfer from an outside institution, time from outside hospital admission to arrival at our institution, time from outside hospital admission to PVAD implantation, duration of IABP support prior to PVAD implantation, whether PCI was performed, baseline cardiac index, and baseline creatinine. Forward and backward step-wise regression were used to test the consistency of the result (threshold $p$-value $<0.05$ ). Cumulative survival was estimated through six months using the Kaplan-Meier method. Thresholds for continuous clinical variables evaluated as predictors of survival were determined using receiver-operator curve analysis.

\section{Results}

\section{Characteristics of the study population}

Between 2006 and 2014 a total of 65 patients underwent TandemHeart PVAD placement at Brigham and Women's Hospital for severe CS. Baseline characteristics of this population are shown in Tables 1-3. Thirty-eight patients (58.5\%) had ICMP and 27 (41.5\%) had NICMP. The majority of patients with NICMP had a dilated type of cardiomyopathy (77.8\%, Figure 1). The most common etiology of NICMP was idiopathic (48.1\%). The majority of patients $(50.7 \%)$ had isolated left ventricular failure, $4.6 \%$ had isolated right ventricular failure, and $44.6 \%$ had biventricular failure (Table 1).

\section{Treatment prior to PVAD support}

A substantial proportion (63.1\%) of patients who progressed to support with a PVAD were transferred from an outside institution, and $43.9 \%$ (18/41) of those patients had initiated MCS (IABP or Impella) prior to transfer. The remainder presented directly to our institution. The median time between presentation to an outside institution and arrival at our institution was 1.7 days (interquartile range (IQR) 0.9-2.9 days). Sixteen patients (24.6\%) had suffered a cardiac arrest during the 48 -hour period preceding PVAD placement. The median time between cardiac arrest and PVAD placement was 9.7 hours (IQR 4.2-17.1 hours). A total of 35 patients $(53.8 \%)$ were treated with IABP counterpulsation prior to PVAD placement. The 
median duration of IABP support in these patients was 11.0 hours (IQR 3.9-33.7 hours; Table 1).

\section{PVAD support}

Of the 65 patients in our analysis, $78.5 \%$ received a left PVAD alone, $7.7 \%$ received a right PVAD alone, and $13.8 \%$ received biventricular PVAD support. The median duration of PVAD support was 5.9 days (IQR 3.7-7.6 days).

\section{Biochemical and hemodynamic parameters}

Baseline laboratory values for the cohort demonstrated substantial end-organ dysfunction prior to PVAD placement, as evidenced by lactic acidosis, renal impairment, and elevated liver transaminases (Table 2). Hemodynamic parameters prior to PVAD implantation indicated severely reduced $\mathrm{CI}$ and elevated left and right heart filling pressures, despite high levels of pharmacologic and mechanical (IABP or Impella) support (Table 3). At 3 hours following PVAD implantation, there was a significant increase in median CI $(p<0.001)$ and MAP $(p=0.032)$, and decreases in RAP ( $p=0.003)$, PCWP $(p=0.001)$, and vasopressor/ inotrope requirement $(p=0.040)$. These hemodynamic improvements were more pronounced at 24 hours following PVAD placement (Figure 2).

\section{Clinical outcomes}

In total, 32 of 65 patients (49.2\%) survived to hospital discharge, and all of these patients remained alive at six months (Figure 3). More than one quarter of patients (26.2\%) received destination surgical therapy, of which five died during the index hospitalization. Of the 12 patients who underwent destination surgical therapy and survived, five underwent surgical left ventricular assist device (LVAD) placement, three patients underwent surgical biventricular assist device placement, two underwent valve surgery, one underwent combination ventricular septal defect repair and valve surgery, and one underwent LVAD placement as a bridge to cardiac transplant during the index hospitalization (Table 4).

Forty-eight patients $(73.8 \%)$ required blood transfusions while on PVAD support, and the median number of PRBC units transfused was 5.0 (IQR 3.0-8.8; Table 1). There were no vascular complications that required surgery or peripheral intervention. Twenty-nine patients had one or more positive blood or central venous catheter tip cultures following PVAD placement, and the most commonly isolated organism (23/29) was coagulasenegative Staphylococcus aureus. In most cases, true bacteremia was not able to be distinguished from contamination.

\section{Clinical parameters associated with survival}

Baseline clinical characteristics associated with survival to hospital discharge (Table 1) included younger age ( $p<0.001)$, NICMP $(p=0.004)$, and lower presenting left ventricular ejection fraction (LVEF) $(p=0.009)$. Survival rates for patients $<45$ years, $45-64$ years, and $\checkmark 65$ years were $86.7 \%, 48.6 \%$, and $13.3 \%$, respectively ( $p$ for trend $<0.001$ ). Type of cardiomyopathy was strongly associated with survival to hospital discharge (70.4\% (19/27) of NICMP patients survived vs 34.2\% (13/38) of ICMP patients; Figure 3). Survival rates for patients with LVEF $₫ 5 \%$, LVEF $16-25 \%$, and LVEF $>25 \%$ were $66.7 \%, 41.2 \%$, and 
$25.0 \%$, respectively ( $p$ for trend=0.010; Figure 4). Notably, none of the baseline hemodynamic and laboratory variables included in this analysis was significantly different between survivors and non-survivors. In a multivariable analysis, younger age $(p=0.026)$ and NICMP (odds ratio (OR) 4.05, 95\% confidence interval (CI) $1.11-14.78 ; p=0.034$ ) remained significant predictors of survival to hospital discharge. These were the only baseline covariates that remained significant in either forward or backwards selection modeling.

Twenty-four hours after PVAD placement, the median change in CI was $+1.10 \mathrm{~L} / \mathrm{min} / \mathrm{m}^{2}$ in survivors and $+0.55 \mathrm{~L} / \mathrm{min} / \mathrm{m}^{2}$ in non-survivors $(p<0.001)$, with a change in vasopressor/ inotrope requirement of -1.0 agents in survivors and 0.0 in non-survivors $(p=0.21)$. Patients with a more modest improvement in CI ( $₫ 0.75 \mathrm{~L} / \mathrm{min} / \mathrm{m}^{2}$ ) had a higher risk of in-hospital mortality (OR 6.3, 95\% CI 1.8-22.1) compared with patients with a greater increase in CI. Considering severity of acidosis, patients with a failure to improve (decrease in serum anion gap $\_$mEq/L) also had higher in-hospital mortality (OR 5.1, 95\% CI 1.6-16.6). In a subset $(n=11)$ of patients with serial serum lactates, mortality was markedly higher in those with a decrease in lactate $\mathcal{s} \mathrm{mmol} / \mathrm{L}$ (Figure 5).

In a sensitivity analysis excluding the 17 patients who underwent a destination surgical intervention during the index hospitalization, the clinical parameters associated with clinical recovery, PVAD explantation, and hospital discharge were unchanged (younger age, NICMP, and lower presenting EF).

\section{Discussion}

There is limited evidence supporting the efficacy of percutaneous MCS for the treatment of severe CS, and even less data regarding appropriate utilization of this resource-intensive technology. Our study of consecutive patients undergoing TandemHeart PVAD implantation over an eight-year period in a large tertiary academic medical center has four major findings. First, PVAD placement resulted in a rapid, significant, and sustained reversal of hemodynamic derangements, and served as a bridge to myocardial recovery in $31 \%$ and definitive surgical therapy in an additional $18 \%$ of patients. Second, despite intensive care in a tertiary academic medical center accompanied by these hemodynamic improvements and destination options, in-hospital mortality in the overall cohort of patients managed with a PVAD remained close to 50\%. Third, we found that survival with this intensive form of support exceeded $70 \%$ in patients with NICMP, with a more favorable survival than in ICMP patients, even after accounting for their generally younger age. Fourth, we found that the early hemodynamic and metabolic response (over 24 hours) may be useful as a guidepost for clinicians and families in considering the likelihood of survival to discharge.

\section{Clinical decision-making regarding PVAD support}

Though the most common cause of CS remains acute myocardial infarction (AMI), nonischemic causes of acute myocardial dysfunction account for an increasing proportion of CS cases. ${ }^{9}$ It is noteworthy that NICMP patients had better outcomes with PVAD support in our analysis. This finding may be a reflection of the natural history of CS and a higher probability of spontaneous ventricular recovery in acute presentations of NICMP as 
compared to ICMP. Alternatively, it may be the case that a treatment strategy of nearcomplete hemodynamic support is better suited to specific NICMP patient populations. Although the finding that younger age is associated with a more favorable prognosis is not surprising, our study provides data to support a general strategy of more aggressive intervention in younger patients. Despite the severity of hemodynamic and metabolic derangement, the $\sim 25 \%$ of patients who were 45 years old or younger had a survival approaching $90 \%$ after PVAD support in our experience.

The finding that lower presenting LVEF was associated with a more favorable prognosis is interesting, if perhaps counterintuitive. One potential interpretation of this result is that for patients whose circulatory failure is driven more by myocardial dysfunction than any other cause, PVAD placement allows for rapid and targeted correction of the underlying process. Appropriate caution must be taken when interpreting this finding, however, given the small sample size of our cohort. In a multivariable analysis controlling for type of cardiomyopathy (NICMP vs ICMP), the relationship between lower presenting LVEF and improved survival was no longer significant. This suggests that this association may also be confounded by the fact that patients with NICMP tended to have both a lower LVEF and a more favorable prognosis.

It is important to note the clinical variables that did not predict survival to hospital discharge in our cohort. Surprisingly, there were no significant differences in baseline laboratory and hemodynamic data between survivors and non-survivors (Tables 1-3). This observation suggests that the severity of a patient's hemodynamic derangements or end-organ dysfunction do not correlate with response to PVAD implantation and should not necessarily affect the decision to initiate PVAD support in patients with CS. Interestingly, however, we found that patients who showed little improvement in hemodynamics or metabolic derangement over the first 24 hours had very poor survival to hospital discharge.

Our findings also lend support to expert opinion that early intervention is important to prevent the downward and complex spiral associated with cardiogenic shock. ${ }^{1}$ Indeed, in our cohort, the duration of IABP support prior to PVAD placement tended to be shorter in survivors compared to non-survivors ( 8.7 hours vs 17.4 hours, Table 1 ). These data suggest that the optimal timing of PVAD placement, particularly in relation to other forms of MCS, deserves further investigation.

\section{Previous studies}

Few data have been published regarding outcomes in patients with CS supported with the TandemHeart PVAD. The best available prospective human data on the use of PVADs in the setting of severe CS comes from a combined meta-analysis of 100 patients with CS, in which left ventricular PVADs were compared with IABPs. ${ }^{10}$ This study included two trials of the TandemHeart device and one trial of the Impella device, an alternative MCS device that is positioned across the aortic valve and provides support by propelling blood from the left ventricle into the aorta. ${ }^{6}$ The meta-analysis demonstrated that, compared with patients receiving IABP counterpulsation, patients receiving left ventricular PVAD support had improved hemodynamics (e.g. MAP, CI) but no difference in 30-day mortality. ${ }^{10}$ 
In the largest published cohort study of patients undergoing TandemHeart PVAD placement for CS $(n=117),{ }^{11} \mathrm{Kar}$ and colleagues similarly observed rapid and significant reversal of hemodynamic and biochemical perturbations. Mortality at 30-days was $40.2 \%$, comparing favorably to historical controls. Our cohort had comparatively better baseline hemodynamic values (median cardiac index 1.9 (IQR 0.86 ) vs 0.5 (IQR 0.8$) \mathrm{L} / \mathrm{min} / \mathrm{m}^{2}$ ), although this may be driven by the $47.9 \%$ of patients in Kar's study who underwent active CPR immediately prior to or during PVAD implantation with an imputed CI of $0.0 \mathrm{~L} / \mathrm{min} / \mathrm{m}^{2}$.

Our findings similarly indicate that PVAD support provides sufficient circulatory support to restore tissue perfusion, bridging the patient to additional decision-making, recovery, or destination options. In younger patients with NICMP, in particular, the possibility of recovery or survival to transplantation likely underlies the near $90 \%$ survival that we observed in our cohort. Notably, it was the majority in this group that survived without progression to transplantation during the follow-up period in our study.

Important complications of the TandemHeart PVAD system include major bleeding, critical limb ischemia, sepsis, tamponade, and residual atrial septal defect. Although there were no vascular complications requiring surgical or percutaneous intervention in our cohort, previous reported estimates of critical limb ischemia necessitating intervention range from $3-33 \% .{ }^{11-13}$ The most commonly reported major bleeding complications are bleeding around the cannula site, groin hematoma, and gastrointestinal bleeding, which occur in 42$90 \%$ of patients, though definitions across studies have been inconsistent. ${ }^{11-13}$ In the Kar et al. study, blood transfusions were needed in $60 \%$ of cases. ${ }^{11}$ Finally, fever and/or sepsis complicating TandemHeart PVAD use have been reported. ${ }^{11-13}$

\section{Limitations}

There are several limitations of our analysis that deserve specific mention. First, as with any cohort study, our findings reflect associations that do not necessarily imply causation.

Second, despite our capture of all consecutive PVAD implantations at our institution over a nearly ten-year period, our statistical power to identify additional clinical predictors, other than those with the strongest associations with survival, was limited by sample size. This limitation highlights the need for collaborative studies of PVAD support as an infrequentlyused but potentially important management option. Third, our analysis is descriptive of outcomes among patients treated with a PVAD. We have not made any inferences with respect to outcomes with PVAD implantation compared with patients with CS managed without PVAD support. There remains a need for randomized trials of PVAD support of sufficient sample size to test the impact on clinical outcomes. Fourth, data were incomplete for some laboratory values (e.g. pre-PVAD lactate, post-PVAD lactate, post-PVAD liver function tests), limiting our ability to assess these variables.

\section{Conclusion}

Younger patients and patients with NICMP who underwent PVAD placement for severe CS had $>70 \%$ survival to hospital discharge. These data provide a basis to help identify patients with CS who have the highest likelihood of survival with PVAD support, and a context for prospective randomized studies of MCS. Our study also showed that patients with less 
robust hemodynamic improvement and persistent metabolic acidosis after 24 hours of PVAD support had very poor survival to hospital discharge. These findings may help to guide clinicians who are tasked with medical decision-making and counseling the family members of these critically ill patients.

\section{Acknowledgments}

\section{Funding}

This quality improvement project was supported by institutional funds and received no specific grant from any funding agency in the public, commercial, or not-for-profit sectors.

\section{References}

1. Reynolds HR, Hochman JS. Cardiogenic shock: current concepts and improving outcomes. Circulation. 2008; 117:686-697. [PubMed: 18250279]

2. Kohsaka S, Menon V, Lowe AM, et al. Systemic inflammatory response syndrome after acute myocardial infarction complicated by cardiogenic shock. Arch Intern Med. 2005; 165:1643-1650. [PubMed: 16043684]

3. Katz JN, Stebbins AL, Alexander JH, et al. Predictors of 30-day mortality in patients with refractory cardiogenic shock following acute myocardial infarction despite a patent infarct artery. Am Heart J. 2009; 158:680-687. [PubMed: 19781431]

4. Thiele H, Allam B, Chatellier G, et al. Shock in acute myocardial infarction: the Cape Horn for trials? Eur Heart J. 2010; 31:1828-1835. [PubMed: 20610640]

5. Thiele H, Zeymer U, Neumann FJ, et al. Intraaortic balloon support for myocardial infarction with cardiogenic shock. N Engl J Med. 2012; 367:1287-1296. [PubMed: 22920912]

6. Naidu SS. Novel percutaneous cardiac assist devices: the science of and indications for hemodynamic support. Circulation. 2011; 123:533-543. [PubMed: 21300961]

7. Stewart GC, Givertz MM. Mechanical circulatory support for advanced heart failure: patients and technology in evolution. Circulation. 2012; 125:1304-1315. [PubMed: 22412091]

8. Ostadal P, Mlcek M, Holy F, et al. Direct comparison of percutaneous circulatory support systems in specific hemodynamic conditions in a porcine model. Circ Arrhythm Electrophysiol. 2012; 5:1202-1206. [PubMed: 23051839]

9. Kar B, Basra SS, Shah NR, et al. Percutaneous circulatory support in cardiogenic shock: interventional bridge to recovery. Circulation. 2012; 125:1809-1817. [PubMed: 22492948]

10. Cheng JM, den Uil CA, Hoeks SE, et al. Percutaneous left ventricular assist devices vs. intra-aortic balloon pump counterpulsation for treatment of cardiogenic shock: a meta-analysis of controlled trials. Eur Heart J. 2009; 30:2102-2108. [PubMed: 19617601]

11. Kar B, Gregoric ID, Basra SS, et al. The percutaneous ventricular assist device in severe refractory cardiogenic shock. J Am Coll Cardiol. 2011; 57:688-696. [PubMed: 20950980]

12. Thiele H, Sick P, Boudriot E, et al. Randomized comparison of intra-aortic balloon support with a percutaneous left ventricular assist device in patients with revascularized acute myocardial infarction complicated by cardiogenic shock. Eur Heart J. 2005; 26:1276-1283. [PubMed: 15734771]

13. Burkhoff D, Cohen H, Brunckhorst C, et al. A randomized multicenter clinical study to evaluate the safety and efficacy of the TandemHeart percutaneous ventricular assist device versus conventional therapy with intraaortic balloon pumping for treatment of cardiogenic shock. Am Heart J. 2006; 152:469.e1-8. [PubMed: 16923414] 


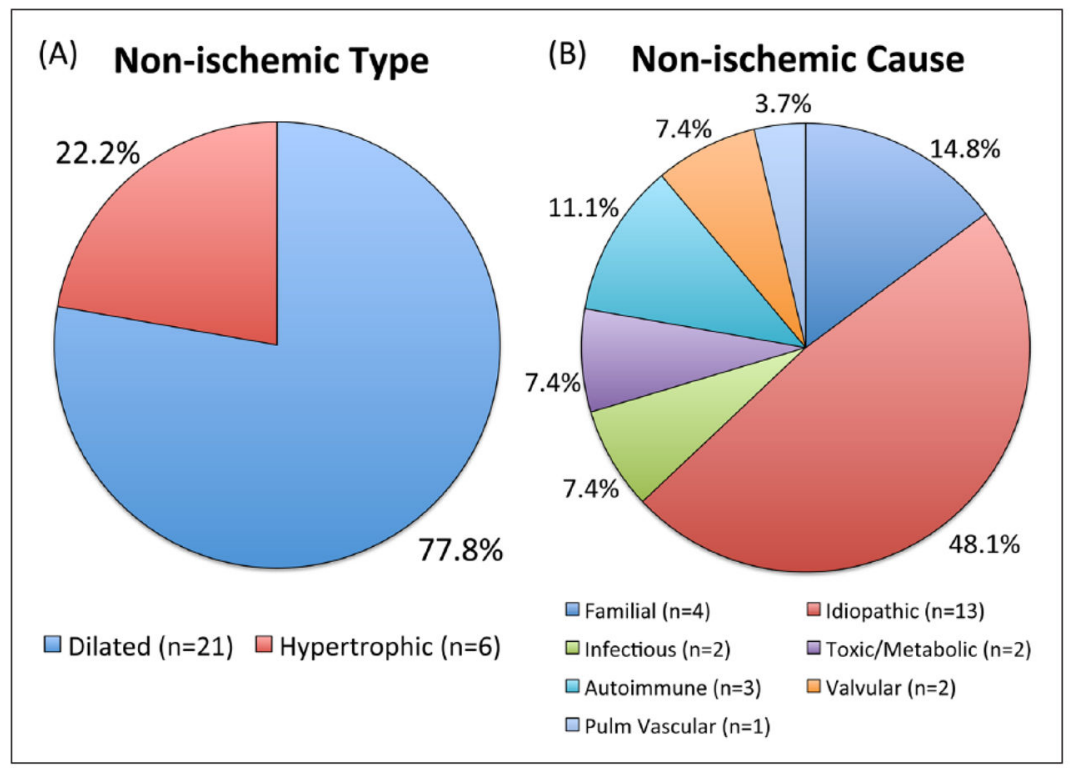

Figure 1.

Classification of non-ischemic cardiomyopathy by type (A) and cause (B). Pulm: pulmonary. 


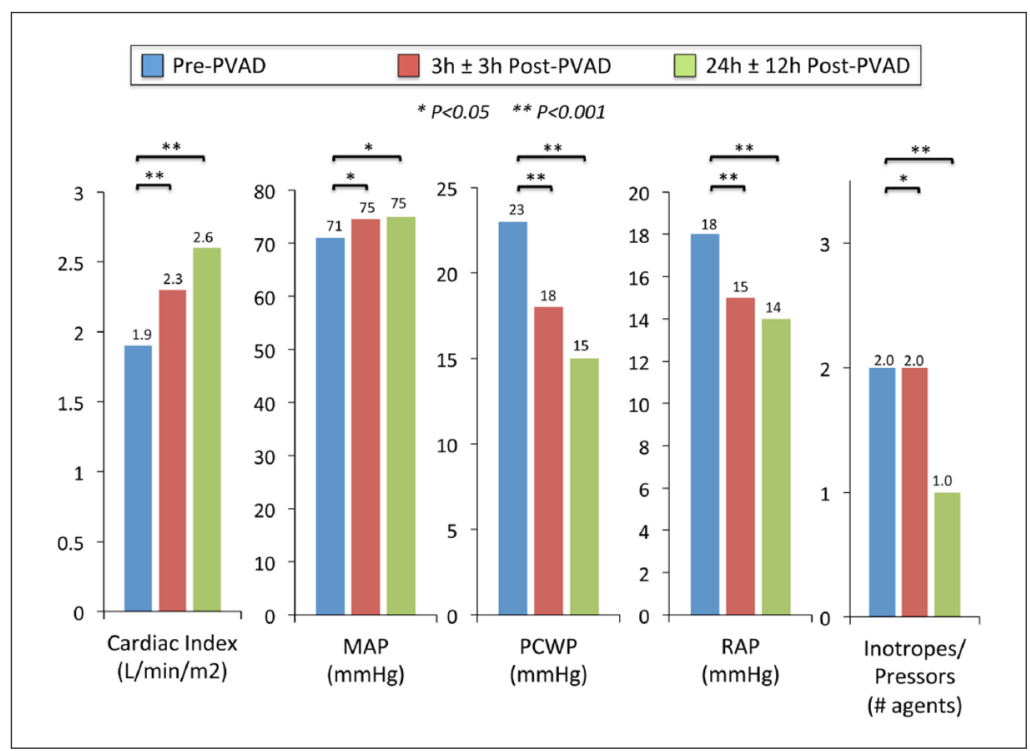

Figure 2.

Hemodynamics prior to percutaneous ventricular assist device placement and at three hours $( \pm 3 \mathrm{~h})$ and 24 hours $( \pm 12 \mathrm{~h})$ after placement. PVAD: percutaneous ventricular assist device; MAP: mean arterial pressure; PCWP: pulmonary capillary wedge pressure; RAP: right atrial pressure. 


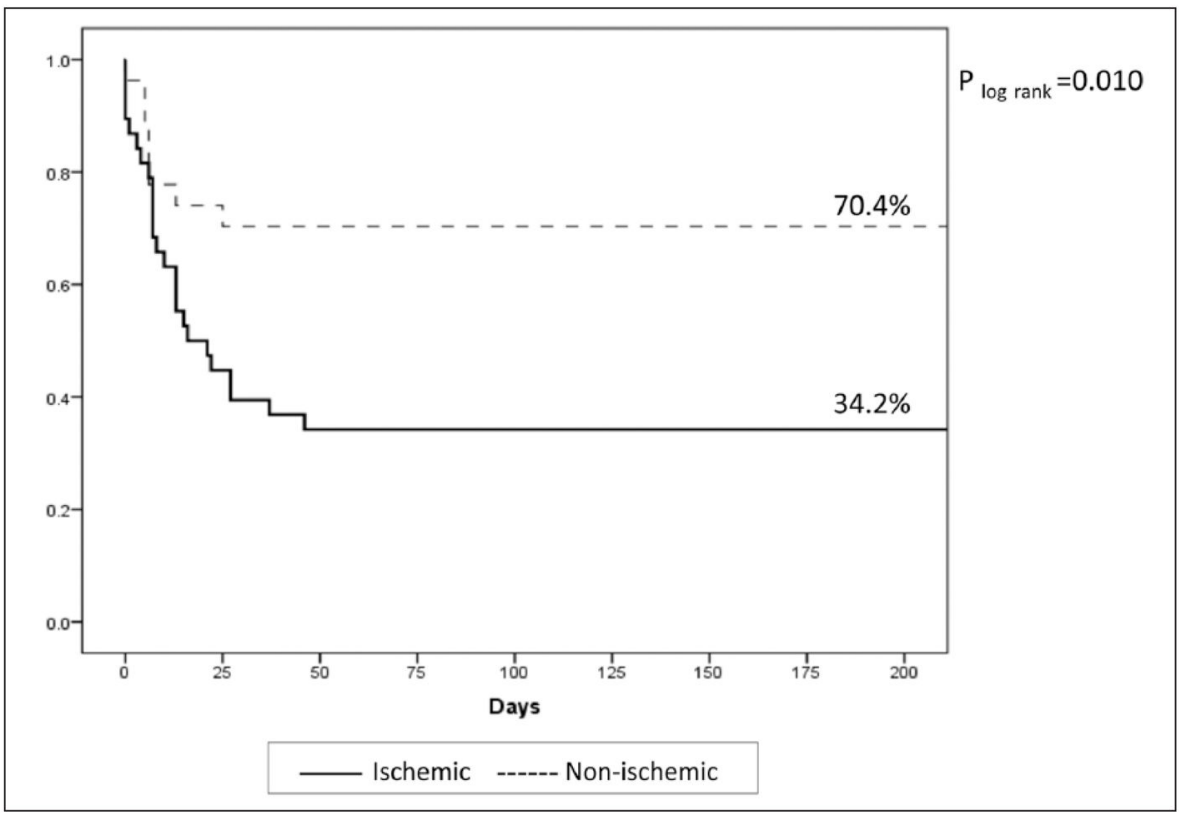

Figure 3.

Kaplan-Meier estimates of survival stratified by ischemic and non-ischemic cardiomyopathy. 


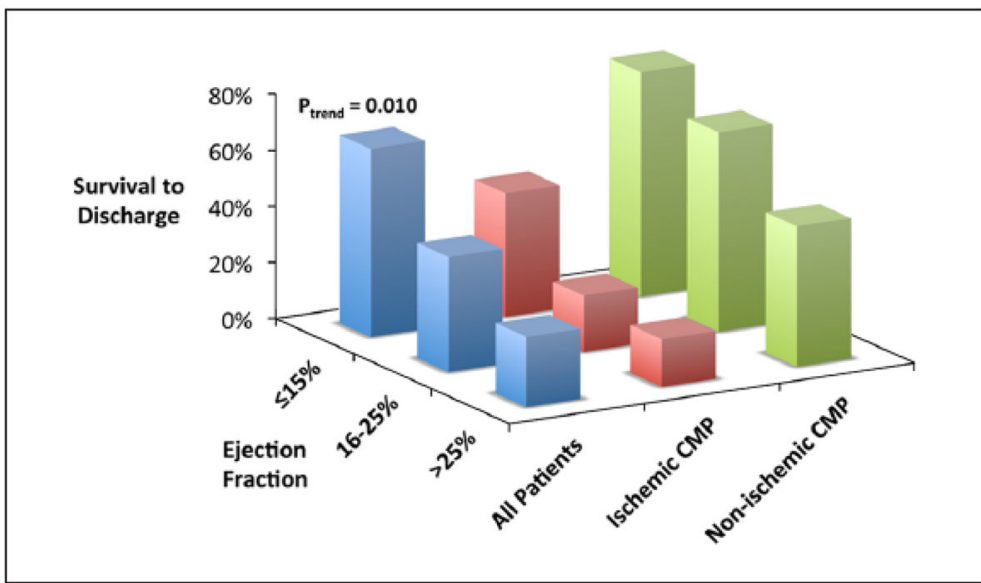

Figure 4.

Survival to hospital discharge stratified by left ventricular ejection fraction and cardiomyopathy type. CMP: cardiomyopathy. 


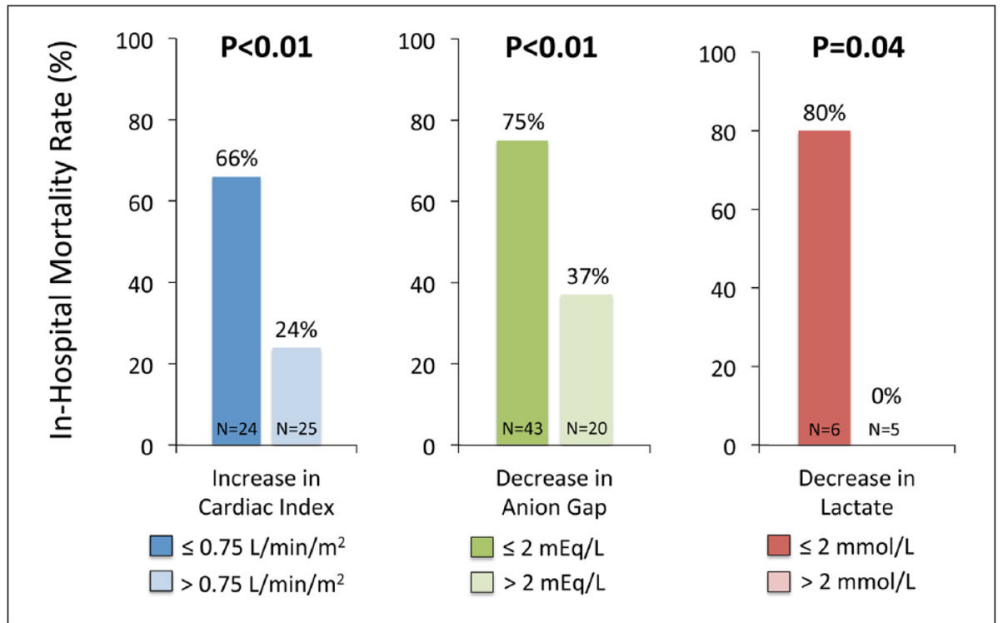

Figure 5.

In-hospital mortality based on the change in cardiac index, lactate, and anion gap 24 hours following PVAD placement. 
Table 1

Clinical characteristics for patients with severe CS undergoing PVAD implantation.

\begin{tabular}{|c|c|c|c|c|}
\hline & All patients $(N=65)$ & Survivors $(N=32)$ & Non-survivors $(N=33)$ & $P$ value \\
\hline Age (years) & $54.1 \pm 14.5$ & $47.3 \pm 14.8$ & $60.6 \pm 10.9$ & $<0.001$ \\
\hline$<45$ years, $n(\%)$ & $15(23.1)$ & $13(40.6)$ & $2(6.1)$ & \\
\hline 45-64 years, $n(\%)$ & $35(53.8)$ & $17(53.1)$ & $18(54.5)$ & $<0.001$ \\
\hline$\Varangle 65$ years, $n(\%)$ & $15(23.1)$ & $2(6.3)$ & $13(39.4)$ & \\
\hline Male (\%) & $51(78.5)$ & $25(78.1)$ & $26(78.8)$ & 0.95 \\
\hline BMI $\left(\mathrm{kg} / \mathrm{m}^{2}\right)$ & $28.7(24.5-33.8)$ & $28.1(24.5-33.8)$ & $29.5(24.2-35.2)$ & 0.40 \\
\hline Pre-PVAD LVEF\% & $20.0(15.0-30.0)$ & $15.0(15.0-20.0)$ & $25.0(15.0-30.0)$ & 0.009 \\
\hline $\mathbf{4 5 \% , n ( \% )}$ & $24(42.1)$ & $16(59.2)$ & $8(26.7)$ & \\
\hline $16-25 \%, n(\%)$ & $17(29.8)$ & $7(25.9)$ & $10(33.3)$ & 0.009 \\
\hline$>25 \%, n(\%)$ & $16(28.1)$ & $4(14.8)$ & $12(40.0)$ & \\
\hline Ischemic CMP, $n(\%)$ & $38(58.5)$ & $13(40.6)$ & $25(75.8)$ & 0.004 \\
\hline Non-ischemic CMP, $n(\%)$ & $27(41.5)$ & $19(59.4)$ & $8(24.2)$ & \\
\hline LV dysfunction, $n(\%)$ & $33(50.8)$ & $14(43.8)$ & $19(57.6)$ & \\
\hline RV dysfunction, $n(\%)$ & $3(4.6)$ & $2(6.3)$ & $1(3.0)$ & 0.52 \\
\hline BiV dysfunction, $n(\%)$ & $29(44.6)$ & $16(50.0)$ & $13(39.4)$ & \\
\hline PCI during hospitalization, $n(\%)$ & $21(32.3)$ & $7(21.9)$ & $14(42.4)$ & 0.091 \\
\hline IABP, n $(\%)$ & $35(53.8)$ & $19(59.4)$ & $16(48.5)$ & 0.44 \\
\hline Impella, $n(\%)$ & $4(6.2)$ & $1(3.1)$ & $3(9.1)$ & 0.61 \\
\hline IABP duration (hh:mm) & $10: 57(3: 56-33: 20)$ & $8: 43(4: 02-15: 33)$ & $17: 22(2: 49-45: 14)$ & 0.30 \\
\hline PVAD duration (dd hh:mm) & $520: 30(3$ 16:46-7 14:29) & $\begin{array}{c}517: 11(321: 13-7 \\
15: 55)\end{array}$ & $523: 05$ (3 02:42-7 15:28) & 0.64 \\
\hline $\begin{array}{l}\text { Cardiac arrest, } n(\%)<48 \text { h prior to } \\
\text { PVAD start }\end{array}$ & $16(24.6)$ & $9(28.1)$ & $7(21.2)$ & 0.47 \\
\hline Time from arrest to PVAD (hh:mm) & $9: 42(4: 10-17: 08)$ & $13: 11(7: 54-25: 46)$ & $4: 44(1: 28-11: 30)$ & 0.18 \\
\hline Patients who underwent RRT, $n(\%)$ & $13(20.0)$ & $4(12.5)$ & $9(27.3)$ & 0.21 \\
\hline $\begin{array}{l}\text { Patients who received PRBC transfusion } \\
\text { on PVAD support, } n(\%)\end{array}$ & $48(73.8)$ & $23(71.9)$ & $25(75.8)$ & 0.72 \\
\hline $\begin{array}{l}\text { Number of PRBC transfusions given while } \\
\text { on PVAD support }\end{array}$ & $5.0(3.0-8.8)$ & $6.0(3.0-10.0)$ & $5.0(3.5-8.0)$ & 0.64 \\
\hline
\end{tabular}

BMI: body mass index; PVAD: percutaneous ventricular assist device; LVEF: left ventricular ejection fraction; CMP: cardiomyopathy; PCI: percutaneous coronary intervention; IABP: intra-aortic balloon pump; RRT: renal replacement therapy; PRBC: packed red blood cells.

Mean \pm SD is presented for normally distributed variables, and median (interquartile range) for variables that are not normally distributed, unless otherwise specified. 


\section{Table 2}

Baseline laboratory values prior to PVAD implantation.

\begin{tabular}{lcccc}
\hline & All patients $(\boldsymbol{N = 6 5})$ & Survivors $(\boldsymbol{N = 3 2})$ & Non-survivors $(\boldsymbol{N = 3 3})$ & $\boldsymbol{P}$ value \\
\hline Sodium, mg/dL & $136(133-139)$ & $136(132-139)$ & $136(134-139)$ & 0.77 \\
Bicarbonate, $\mathbf{~ m g / d L}$ & $21.0(17.0-22.0)$ & $19.5(17.0-22.0)$ & $21.0(16.0-24.0)$ & 0.25 \\
Anion gap & $13.0(10.0-17.0)$ & $13.0(10.3-17.5)$ & $13.0(10.0-17.0)$ & 0.73 \\
Lactate, $\mathbf{~ m m o l / L ~}$ & $3.6(1.9-7.6)$ & $4.6(1.8-8.5)$ & $3.2(2.0-4.0)$ & 0.31 \\
Arterial pH & $7.34(7.22-7.43)$ & $7.32(7.18-7.40)$ & $7.37(7.30-7.44)$ & 0.24 \\
BUN, mg/dl & $31.0(22.0-49.5)$ & $28.0(20.5-38.5)$ & $37.0(25.5-57.5)$ & 0.06 \\
Creatinine, mg/dL & $1.8(1.2-2.7)$ & $1.6(1.1-2.7)$ & $1.9(1.3-2.7)$ & 0.35 \\
ALT, U/L & $177(57-641)$ & $206(71-1131)$ & $152(50-319)$ & 0.18 \\
AST, U/L & $274(86-889)$ & $369(163-3707)$ & $267(63-547)$ & 0.11 \\
Total bilirubin, mg/dL & $1.0(0.6-2.2)$ & $0.9(0.6-1.9)$ & $1.1(0.5-3.4)$ & 0.52 \\
INR & $1.5(1.3-2.8)$ & $1.6(1.2-2.8)$ & $1.5(1.3-1.8)$ & 0.58 \\
Platelets & $211(158-298)$ & $231(158-290)$ & $200(134-311)$ & 0.66 \\
\hline
\end{tabular}

BUN: blood urea nitrogen; ALT: alanine aminotransferase; AST: aspartate aminotransferase; INR: international normalized ratio. Note: Variables are presented as medians (interquartile range). 
Table 3

Baseline hemodynamic values prior to PVAD implantation.

\begin{tabular}{lcccc}
\hline & All patients $(\boldsymbol{N = 6 5})$ & Survivors $(\boldsymbol{N = 3 2})$ & Non-survivors $(\boldsymbol{N = 3 3})$ & $\boldsymbol{P}$ value \\
\hline Cardiac index, $\mathbf{L} / \mathbf{m i n} / \mathbf{m}^{\mathbf{2}}$ & $1.9(1.5-2.4)$ & $1.8(1.5-2.1)$ & $2.1(1.7-2.6)$ & 0.08 \\
Mean arterial pressure, $\mathbf{m m H g}$ & $71(63-78)$ & $73(64-79)$ & $69(63-76)$ & 0.16 \\
Right atrial pressure, $\mathbf{m m H g}$ & $18(15-21)$ & $19(15-21)$ & $16(15-21)$ & 0.32 \\
PCWP, $\mathbf{m m H g}$ & $24(20-30)$ & $27(21-30)$ & $23(18-31)$ & 0.59 \\
Pressors/inotropes, \# agents & $2.0(1.0-3.0)$ & $2.0(1.0-3.0)$ & $2.0(1.0-3.0)$ & 0.34 \\
\hline
\end{tabular}

PCWP: pulmonary capillary edge pressure.

Note: Variables are presented as medians (interquartile range). 


\section{Table 4}

Destinations of survivors to hospital discharge stratified by cardiomyopathy type.

\begin{tabular}{lcc}
\hline & Ischemic $(N=\mathbf{1 3})$ & Non-ischemic $(N=\mathbf{1 9})$ \\
\hline Recovery & 7 & 13 \\
Surgical LVAD & 2 & 3 \\
Surgical BiVAD & 2 & 1 \\
Valve surgery & 1 & 1 \\
LVAD/transplant & 0 & 1 \\
VSD/valve surgery & 1 & 0 \\
\hline
\end{tabular}

LVAD: left ventricular assist device; BiVAD: biventricular assist device; VSD: ventricular septal defect. 